\title{
High-power fiber laser based on a non filamented-core fully-aperiodic large pitch fiber
}

\author{
A. Benoit*a, R. Dauliat ${ }^{\mathrm{a}}$, D. Darwich ${ }^{\mathrm{a}}$, R. Jamier ${ }^{\mathrm{a}}$, S. Grimm $^{\mathrm{b}}$, Jens Kobelke ${ }^{\mathrm{b}}$, K. Schuster $^{\mathrm{b}}$ and \\ P. Roy ${ }^{\mathrm{a}}$ \\ ${ }^{a}$ Univ. Limoges, CNRS, XLIM, UMR 7252, F-87000 Limoges, France \\ ${ }^{\mathrm{b}}$ Leibniz Institute of Photonic Technology, Albert-Einstein-Straße 9, 07745 Jena, Germany
}

\section{EXTENDED ABSTRACT}

Since the double-clad fiber architectures development, fiber-based lasers have witnessed an impressive power scaling [1]. The rise of the extracted signal power has been accompanied by the development of Very Large Mode Area (VLMA) fiber designs allowing the overtake of some key hurdles like the non-linear process or photo-darkening [2]. However, due to a significant deposition of heat along and across the section of VLMA fibers, a new phenomenon, referring to as thermal-induced modal instabilities, has been evidenced recently as being the current limitation that hampers any further power increase in the field of high beam quality fiber lasers [3]. Much of a work has been done by researchers so as to clarify the origin of such detriment, leading to the evidencing of a thermal-induced long-period gratings that enable for an effective coupling of the fundamental mode with an unwanted high-order core mode (typically the LP11) [4].

In order to push away the appearance power threshold of this limitation, new aperiodic cladding microstructurations have been proposed to improve the higher-order modes (HOM) rejection and delocalization out of the gain region. In such way, a truly singlemode operation can be achieved while the energy transfer toward high-order modes is inhibited as long as the LP11 is not confine properly into the fiber core, situation which should occur for heat load power level twice higher than into standard air-silica LPF [5]. These aperiodic microstructures, called fully-aperiodic large pitch fibers (FA-LPF), have proved recently their potential to provide an efficient HOM delocalization while preserving a strong confinement of the fundamental mode into the core of passive VLMA structures [6]. Additionally to this concept of aperiodic cladding structures, we proposed to produce a high index fully doped gain material that is homogeneous, breaking by the way with the current method for synthetizing the active core of VLMA fibers. Then one can obtain a high rare earth concentration over the whole core region whereas conventional CVD cores are diluted with low index passive materials for achieving a perfect index matching with the pure silica. Thus, to ensure a singlemode behavior, the real challenge is to provide a perfect matching between the refractive indices of the high index materials constituting the core region and the cladding background. The aperiodic structure is then made of a set of judiciously distributed low index passive silica inclusions with respect to the aperiodic cladding pattern designed numerically. So as to reach this aim, we have used several silica fabricated using the REPUSIL fabrication process. This method is based on the sintering and vitrification of doped-silica micrometric powders. Thanks to it, it is possible to produce large volume of homogeneous high purity doped silica with a high level of control on the refractive index (about $1.10^{-4}$ ).

For this first demonstration in laser operation, an Yb-doped solid fully-aperiodic large pitch fiber has been manufactured by the REPUSIL process. This fiber has a core diameter of $40 \mu \mathrm{m}$ (average measures of dimensions between low-index inclusions surrounding the active core) corresponding to a mode field area of $910 \mu \mathrm{m}^{2}$, an air-clad diameter of $210 \mu \mathrm{m}$ and an outer diameter of $1.2 \mathrm{~mm}$. A $90 \mathrm{~cm}$-long piece of fiber has been placed in a simple laser cavity. The laser cavity was formed by using a high reflectivity mirror at $1030 \mathrm{~nm}$ and the $4 \%$ Fresnel reflection due to the polished end facet at $0^{\circ}$. A schematic representation of the experimental setup is represented in figure 1.a. 


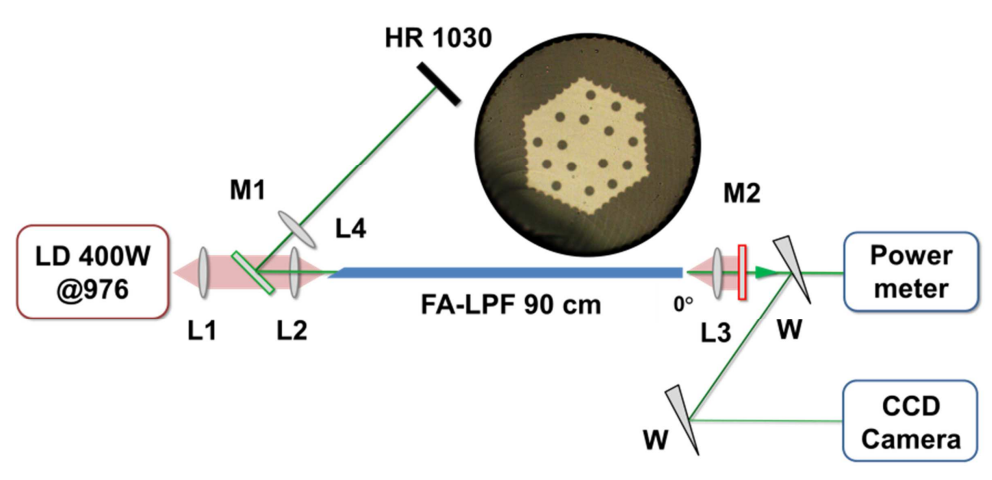

(a)

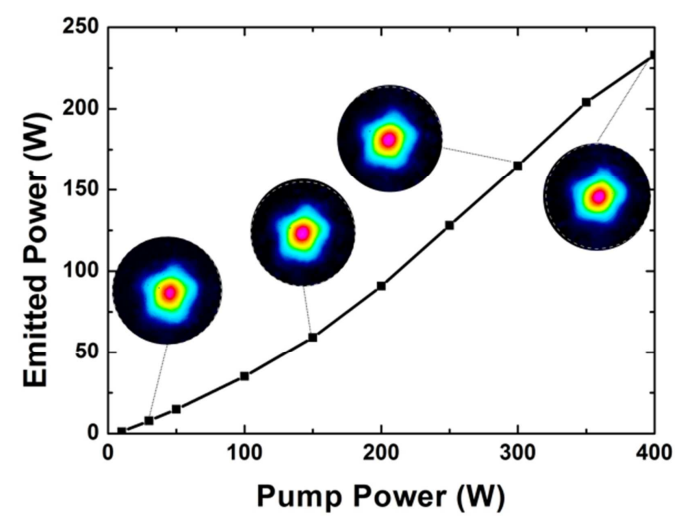

(b)

Figure 1. (a) Schematic representation of experimental set-up. HR 1030 high reflectivity mirror at $1030 \mathrm{~nm}$; Dichroic mirror, M1 (HR1030 / HT976) and M2 (HR976 / HT1030); L1, L2, L3 and L4 are anti-reflection coating collimating and focusing lenses W, wedge plate. Inset microscope image of the fully-aperiodic LPF. (b) Evolution of the extracted power versus the pump power. In insert: the near diffracted beam intensities at the $7.8 \mathrm{~W}, 59.2 \mathrm{~W}, 165 \mathrm{~W}$ and $233 \mathrm{~W}$.

In this configuration, we have emitted an average output power of $233 \mathrm{~W}$ for an available pump power of $400 \mathrm{~W}$ corresponding to a measured slope optical-to-optical efficiency of $58 \%$ represented in figure 1.b. The evolution of the near field intensity distributions is also represented in insets of the figure 1.b. One can observe the absence of beam degradation during this rise of power and a singlemode fashion. Moreover using a commercial $\mathrm{M}^{2}$ measurement device, the preliminary $\mathrm{M}^{2}$ measurements have shown a full power output beam close to the near diffraction limited beam quality, having $\mathrm{M}^{2}$ values less than 1.4. All the beam characterizations and developments to this fully-aperiodic large pitch fiber will be shown during the conference.

To conclude we report on the first high power emission demonstration obtained using a solid non-filamented core fully-aperiodic large pitch fiber manufactured by the REPUSIL method. Using a simple laser cavity, an average output power of $233 \mathrm{~W}$ has achieved for the first time in such a fiber. The preliminary $\mathrm{M}^{2}$ have shown values less than 1.4 , close to near diffraction limited beam quality.

\section{REFERENCES}

[1] M.N. Zervas et al., IEEE Journal of selected topics in Quantum Electronics, 20, 5, 2014

[2] T. Eidam et al., Opt. Exp. vol. 19, n ${ }^{\circ} 1$, pp 255-260, 2010

[3] T. Eidam et al., Opt. Exp. vol. 19, n¹4, pp 13218-13224, 2011

[4] C. Jauregui et al., Opt. Exp. vol. 20, n ${ }^{\circ}$, pp 12912-12925, 2012

[5] R. Dauliat et al., Opt. Exp. vol. 21, n¹6, pp 18927-18936, 2013

[6] A. Benoît et al., Opt. Lett, vol. 39, n 15, pp 4561-4564, 2014 